\title{
DEVELOPMENTS IN CHROMATO-POLAROGRAPHY
}

\author{
WikTOR KeMULA \\ Institute of Physical Chemistry, Polish Academy of Science, \\ Warsaw, Poland
}

\begin{abstract}
Developments in chromato-polarography and the results obtained from its application to the analysis of mixtures of organic compounds are presented. The possibility of determining traces of organic impurities in organic substances by this technique is evaluated.
\end{abstract}

The polarographic method is today one of the most important instrumental methods and has found wide application in analytical chemistry both of inorganic salts and also of organic compounds. When mixtures of different inorganic or organic compounds are present in the solution under test and have to be quantitatively evaluated, the problem is sometimes very difficult and complicated to solve, especially when there are present several ions or substances having similar electrochemical properties, i.e. the same or nearly the same half-wave reduction or oxidation potentials.

When inorganic ions with the same or similar half-wave potentials are present, the problem can be quite easily solved by introducing complexing agents, which shift correspondingly the half-wave potentials of analysed substances, forming complexes of different stability. This procedure is usually not applicable in the case of mixtures of similar organic compounds, because they usually do not form analogous complexes having different electrochemical properties.

This basic difficulty of polarography is especially important when homologues or isomers are present in the mixture, because such substances usually show identical or similar half-wave potentials.

To solve the problem of quantitative separation and analysis of such mixtures it was necessary first to perform a preliminary separation of species and then perform polarographic analysis of these species.

The way we have chosen was chromatographic separation using a 'liquid chromatogram' method combined with continuous polarographic detection of species eluted from the chromatographic column.

Chromato-polarography is a method which combines the high sensitivity and other excellent properties of the dropping mercury electrode as detector -such as the linear relationship between the diffusion current and the concentration of reducible substances--with the high resolving power of the mixtures on the chromatographic column ${ }^{1}$.

When the chromatographic column is filled with an adsorbent (e.g. alumina or an ion-exchange resin) characterized by a non-linear adsorption 
isotherm, 'tails' are usually observed on the elution curve, hindering the complete separation of the components of the mixture to be analysed. To avoid this difficulty we used columns filled with powdered rubber, swollen with organic solvents, which form the immobile phase. Such columns have 'inverse' partition chromatographic functions, and-what is more important-we have stated the fact that when using the supporting electrolyte as a percolating liquid, the straight form of the partition isotherms - when the concentration of the neutral salt is great enough-occurs. In this way, a more favourable separation of substances can be achieved and the 'tails' are diminished or may disappear. Complex salts forming clathrates as a filler are also a very successful separating system ${ }^{2}$.

We have stated that the 'reversed phase' partition system is very successful in the separation of homologues; it separates series of analogous molecules differing by the molecular weight ${ }^{3}$, when the clathrate-forming complex salts separate extremely selectively isomers--molecules differing in shape ${ }^{2}$.

A scheme of a typical chromato-polarographic arrangement has been published earlier ${ }^{1}$. Methods of preparing the separating systems used in chromato-polarography have also been published previously ${ }^{4}$.

Quantitative evaluation of the recorded chromato-polarograms can usually be made by two methods ${ }^{3}$ : elution, and a frontal one.

More precise results can be obtained by the second (frontal) method, but it does require a larger sample for analysis.

The accuracy obtained by the elution method $^{3}$ is sufficient; one analysis lasts two to three hours.

Using partition systems, many factors play an important role. For example, acidity plays a decisive role $^{5}$ when substances to be separated are weak electrolytes. By changing the acidity of the percolating liquid-the moving phase--even the order of elution of different isomers can be reversed. This fact can be very important when one of the separated substances is many times less concentrated than other substances. Also the presence of organic solvents in the moving phase plays a very important role and is widely applied.

Using the simple device described we succeeded in separating and analysing many mixtures of isomers and homologues, e.g. nitroanilines ${ }^{6}$, chloronitrobenzenes ${ }^{6}$, and nitrophenols ${ }^{13}$, mono-, di- and tri-nitrobenzoic acids, nitroalkanes series ${ }^{7}$, nitrofuraldoximes ${ }^{8}$, isomers of $\mathrm{DDT}^{9}$, products of the hydrogenation of diethylbenzene ${ }^{10}$ etc.

Not only partition systems can be used for separation.

Using ion-exchangers as column fillers we were able by a 'salting out' procedure to separate nitroalcohols, nitrobenzoic acids, and nitroalkanes ${ }^{11}$.

During numerous trials of separation of mixtures of different organic molecules we met with obstacles. For example we could not separate by the reversed phase partition method mixtures of several aliphatic and aromatic isomers.

We were able, however, to separate these mixtures first by applying--as column fillers---clathrate-forming complex salts of the $\mathrm{Ni}(\mathrm{CNS})_{2} /(\gamma-$ picoline) ${ }_{4}$ type $^{2,3}$.

For example, mixtures impossible to separate by partition systems have 


\section{DEVELOPMENTS IN CHROMATO-POLAROGRAPHY}

been those of isomers of nitropropane and nitrobutane ${ }^{12}, \alpha^{-}$and $\beta$-nitronaphthalenes ${ }^{14}, \alpha$ - and $\beta$-methylnaphthalenes ${ }^{15}$, syn- and anti-furaldoximes $^{16}$, mononitrotoluenes ${ }^{17}$, and mononitroethylbenzenes ${ }^{18}$. Cis- and trans-azobenzenes, nitroxylenes etc. could be quite easily separated when clathrate-forming compounds were used as column fillers.

As a result of our investigations we can, in general, state that partition chromatography is especially suited for the separation of homologues--. molecules differing in molecular weight, while clathrates are excellent for the separation of isomers-molecules differing by molecular shape.

This statement led us to the investigation of partition coefficients of both systems separately and of mixtures of both separating systems in general. We have published many papers concerning these problems (see literature).

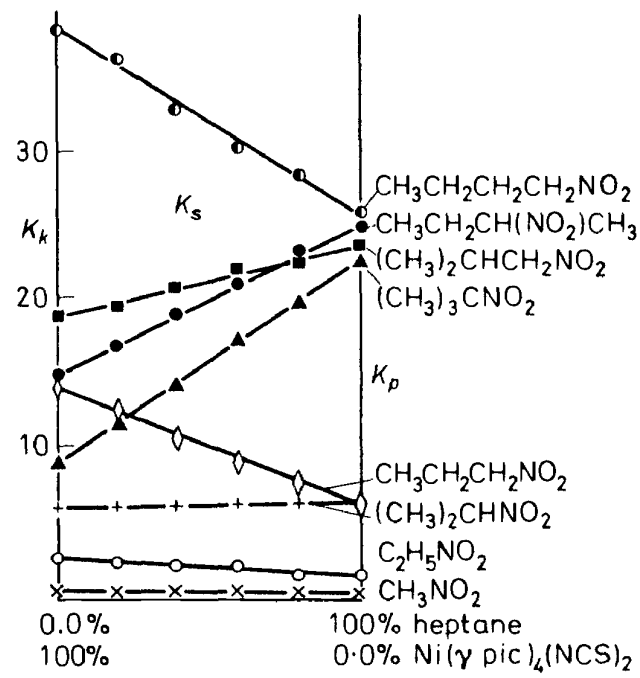

Figure 1. Partition coefficients of pure 'reversed phase' partition $K_{p}$ and clathrate-forming complex-salts systems $K_{k}$ and their complex mixtures.

Based on the results obtained of investigations of partition equilibria on different systems one of our main goals was to prepare one complexseparating system with variable properties of partition according to the molecular weight and shape of the molecules.

Thorough study of the properties of such a combined system led us successfully to the preparation of a complex filler which enabled complete quantitative separation of eight nitroaliphatic species from $C_{1}$ to $C_{4}$ in one experiment $t^{19}$.

One of the most important problems of modern chemistry is trace analysis, especially trace analysis of impurities in organic substances.

Not long ago the $2 \mathrm{~N}(99 \%)$ or $3 \mathrm{~N}(99.9 \%)$ degree of purity of organic substances was used for industrial purposes. Nowadays degrees of purity may be as high as $4 \mathrm{~N}$ or $5 \mathrm{~N}$ and even $6 \mathrm{~N}$ or $7 \mathrm{~N}$.

Progress in the analytical evaluation of very small quantities of organic impurities is not great.

As examples of possibilities of the chromato-polarographic method for 
WIKTOR KEMULA

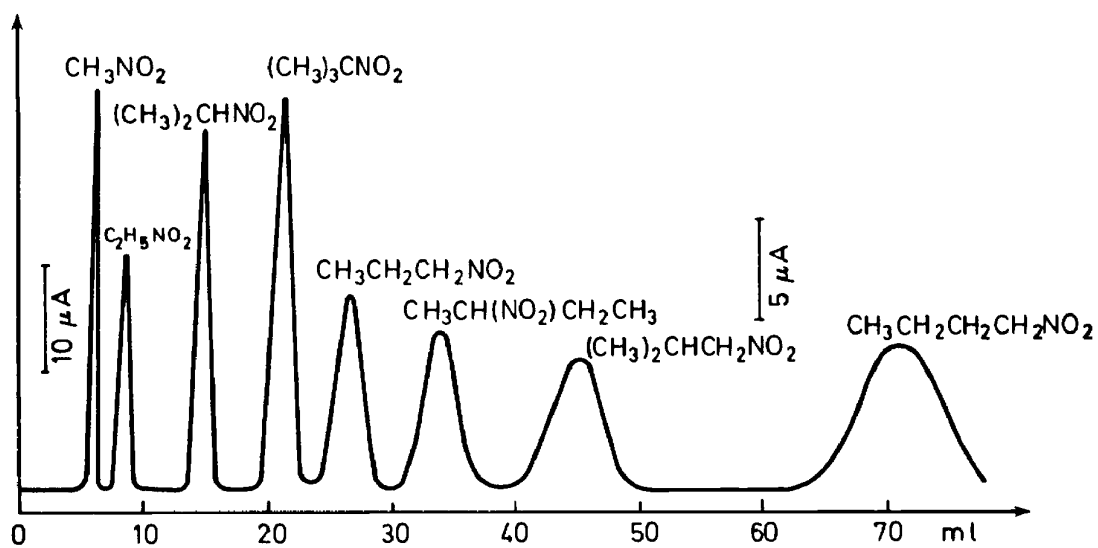

Figure 2. The complete separation of the mixture of eight nitroalkanes $C_{1}$ to $C_{4}$ on complexseparating systems.

analysis of trace amounts can be mentioned here the detection and quantitative evaluation of traces of 2,3-dinitrotoluene and 2,4,6-trinitrotoluene in mixtures of dinitrotoluenes ${ }^{20}$. Using chromato-polarographic separation on $\mathrm{Ni}(\mathrm{NCS})_{2}(\gamma \text {-picoline })_{4}$ with $\gamma$-picoline as a column filler, we were able to separate $o^{-}, m^{-}, p$-nitrotoluenes, $2,3-; 3,5-; 3,4-; 2.4-; 2,6-; 2,5$-dinitrotoluenes and carry out trace analysis of 2,4,6-trinitrotoluene and 2,3dinitrotoluene in a 2,4-and 2,6-dinitrotoluene mixture. Using a new detection

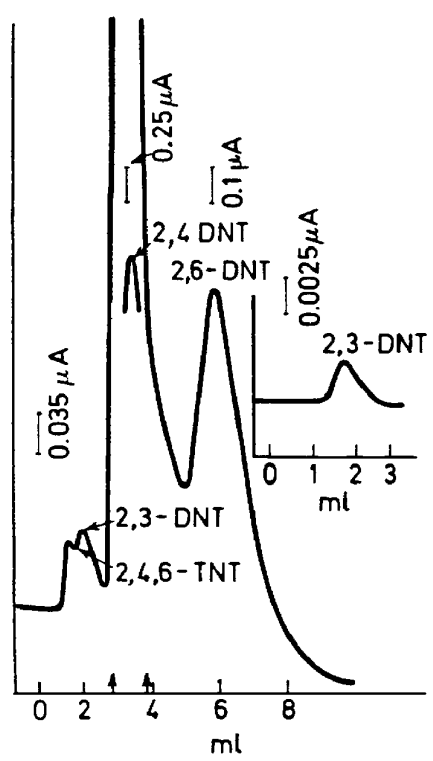

Figure 3. The trace analysis of mononitrotoluenes, 2,4,6-TNT and 2,3-DNT in technical DNT (main diagram). Traces of 2,3-DNT in a special detection set (see inset). 


\section{DEVELOPMENTS IN CHROMATO-POLAROGRAPHY}

set enabling us to de-aerate the percolating liquid, very small amounts of 2,3-dinitrotoluene could be detected.

In these experiments only a $7 \mathrm{~cm}$ long and $0.5 \mathrm{~cm}$ wide column has been used. The weight of the sample analysed was $84 \mu \mathrm{g}$ of dinitrotoluene (technical grade purity) in $0.1 \mathrm{ml}$ of the solution. The detected amounts have been $0.3 \mu \mathrm{g}$ down to $0.03 \mu \mathrm{g}$ of the impurity. Slight 'tailing' observed on the recorded curves was caused by the volume of the detecting vessel being too large. The amount of $0.006 \%$ of impurities in the analysed dinitrotoluene could be evaluated ${ }^{17}$ (See Figure 3).

So far, we have discussed the possibilities of separating substances reducible at the dropping mercury electrode, but it was also possible to extend the application of chromato-polarography to the detection of polarographically inactive substances.

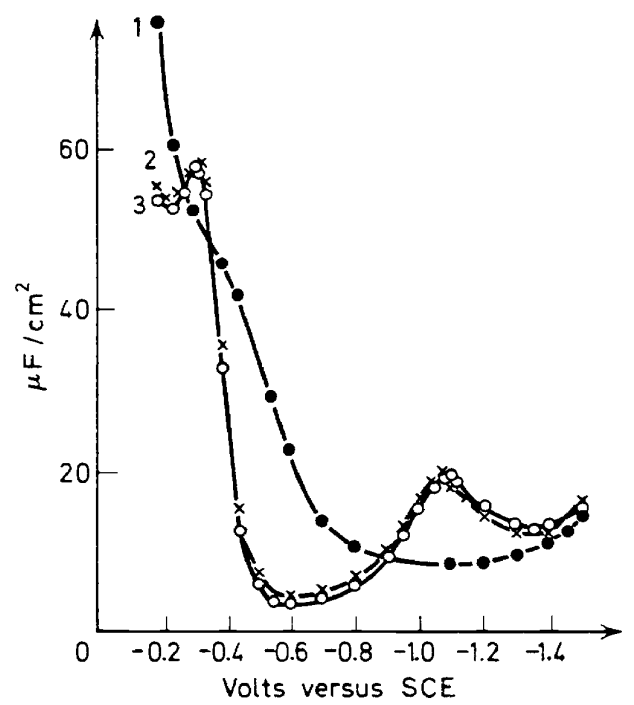

Figure 4. Changes of double-layer capacity of the dropping mercury electrode measured by the a.c. method (1), after addition of $\alpha-(2)$, and $\beta$-nitronaphthalenes (3).

Thus, the surface activity of some organic substances--especially those of high molecular weight-can be utilized for separation by chromatopolarography of some molecules not reducible on the dropping mercury electrode. As an example of a separation of this kind, an analysis of a mixture of brucine and strychnine ${ }^{21}$, based on the suppressive action of these molecules on the polarographic maxima, can be quoted, enabling detection when catalytic maxima of these alkaloids are used ${ }^{21}$.

Two further examples of the activation of polarographically inactive substances can be given. The amino acids are polarographically inactive, but form polarographically reducible complex molecules with copper phosphate ${ }^{22}$. Simply by passing the percolating liquid through a short 


\section{WIKTOR KEMULA}

glass tube filled with solid copper phosphate, the chromato-polarographic detection of amino acids has been achieved.

Another more general way we have chosen in recent years is to use a.c.polarography, which enables us to detect easily in an eluate the presence of organic molecules which usually are surface active ${ }^{23}$.

For extremely small quantities of substances to be separated a special differential method has been elaborated ${ }^{24}$. Giving the above-mentioned examples of chromato-polarographic analysis one can have an idea of the applicability of chromato-polarography to the analysis of complex mixtures of organic substances, which cannot be carried out using conventional polarography.
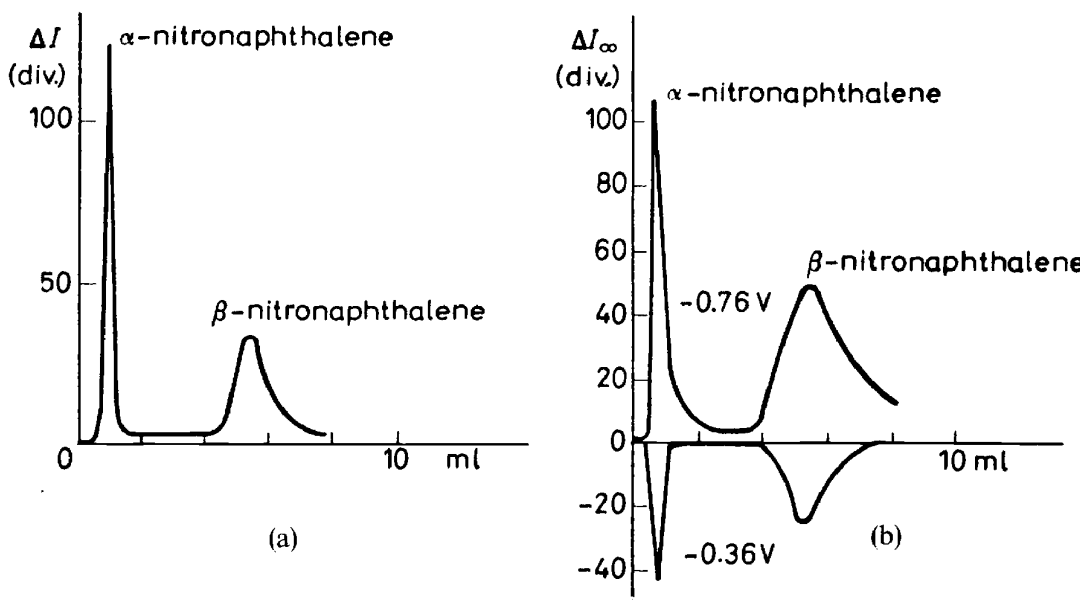

Figure 5. Separation of $\alpha$ - and $\beta$-nitronaphthalenes by d.c. (curve a) and a.c. (curves b) detection methods (compare Figure 4).

Chromato-polarography enables us not only to perform the analyses but also to separate the very minute quantities of the different fractions of partitioned substances by collecting them in separate vessels. These fractions may be investigated further by conventional polarography or other methods.

The problem of separation of mixtures of organic compounds has been shown here partially as a problem of the separation of homologues, with isomers as more difficult examples of separation using complex compound forming clathrates. Frequently the more easily realized reversed phase partition chromatography system is sufficient for solving analytical problems. Various other mixtures-not mentioned here--have been separated and the results published.

We have presented here only the results of the analytical application of chromato-polarography. No purely physicochemical results concerning the mechanism of the separation properties of clathrate-forming complex salts, thermogravimetric and other properties have been mentioned here. 


\section{DEVELOPMENTS IN CHROMATO-POLAROGRAPHY}

Most recently we succeeded in applying absorption spectrophotometry with continuous fast recording of the spectra of eluted substances instead of the dropping mercury electrode. Such a combination is very promising and enabled us to detect, identify and evaluate the nature and amount of separated substances. The results will be published soon ${ }^{25}$.

We still continue also the basic work on the properties of separating systems and apply the obtained results for the solution of practical problems.

\section{REFERENCES}

${ }^{1}$ W. Kemula, Roczn. Chem. 26, 281, 639 and 696(1952).

W. Kemula, Przemysi Chem. 33 (10), 453 (1954).

W. Kemula and A. Górski, Roczn. Chem. 26. 639 (1952).

W. Kemula and A. Górski, Progress in Polarography, Vol. II, p 397. Interscience: London (1962).

W. Kemula and A. Górski, Chemia Anal., Warsaw, 13, 1013 (1968).

2 W. Kemula and D. Sybilska, Nature, London, 185, 237 (1960).

W. Kemula and D. Sybilska, Acta Chim. Acad. Sci. Hung. 27, 137 (1961).

3 W. Kemula, D. Sybilska and J. Geisler, Roczn. Chem. 29, 643(1955).

W. Kemula, D. Sybilska and J. Geisler, Chemia Anal., Warsaw, 1, 144 (1956).

W. Kemula and D. Sybilska, Chemia Anal., Warsaw, 4, 123 (1959).

4 W. Kemula, D. Sybilska and K. Butkiewicz, Roczn. Chem. 39, 61 (1965).

5 W. Kemula, K. Butkiewicz, J. Geisler and D. Sybilska, Chemia Anal., Warsaw, 1, 158 (1956).

6 W. Kemula, D. Sybilska and J. Geisler, Chemia Anal., Warsaw, 1. 144 (1956).

7 W. Kemula and D. Sybilska, Chemia Anal., Warsaw, 4, 123 (1959).

W. Kemula and D. Sybilska, Acta Chim. Acad. Sci. Hung. 27, 137 (1961).

${ }^{8}$ W. Kemula, D. Sybilska and K. Chlebicka, Revue de Chimie, Académie de la Republique Populaire Roumaine, 7, 1003 (1962).

W. Kemula, D. Sybiliska and K. Chlebicka, Roczn. Chem. 39, 1499 (1965).

9 W. Kemula and A. Krzemińska, Chemia Anal., Warsaw, 5, 611 (1960).

${ }_{10}$ W. Kemula, J. Paściak and D. Sybilska, Chemia Anal., Warsaw, 6, 807 (1961).

11 W. Kemula and S. Brzozowski, Roczn. Chem. 35, 703 (1961).

12 W. Kemula and D. Sybilska, Acta Chim. Acad. Sci. Hung. 27, 137 (1961).

${ }^{13}$ W. Kemula, K. Leśniak and D. Sybilska, Proceedings of the International Symposium on Nitrocompounds (Warsaw), p 53. Pergamon: Oxford (1963).

$\mathrm{K}$. Leśniak and T. Urbański, Proceedings of the International Symposium on Nitrocompounds (Warsaw), p 61. Pergamon: Oxford (1963).

14 W. Kemula, D. Sybilska and K. Duszcyk, Microchem. J. 11, 296 (1966).

15 W. Kemula, B. Behr, K. Chlebicka and D. Sybilska, Roczn. Chem. 39,1315(1965).

16 W. Kemula, D. Sybilska and K. Chlebicka, Revue de Chimie, Académie de la République Populaire Roumaine, 7, 1003 (1962).

17 W. Kemula and K. Butkiewicz, Roczn. Chem. 39, 73 (1965).

W. Kemula, D. Sybilska and A. Kwiecińska, Roczn. Chem. 39, 1101 (1965).

18 W. Kemula and D. Sybilska, Analyt. Chim. Acta, 38, 97 (1967).

19 W. Kemula and D. Sybilska, Roczn. Chem. 38, 861 (1964).

20 W. Kemula, A. Kurjan and A. Kwiecińska, Chemia Anal., Warsaw, 12, 869 (1967).

W. Kemula, A. Kwiecińska and D. Sybilska, III Symposium polskoczechoslowackie petroi karbo-chemiczne, Novaky (1967), p 97. Instytut Chemii Ogólnej: Warsaw (1967).

21 W. Kemula and Z. Stachurski, Roczn. Chem. 30, 1285 (1956).

22 W. Kemula and J. Witwicki, Roczn. Chem. 29, 1153(1955).

23 W. Kemula, B. Behr, Z. Borkowska and J. Dojlido, Coll. Czech. Chem. Commun. 30, 4050 (1965).

W. Kemula, B. Behr, K. Chlebicka and D. Sybilska, Roczn. Chem. 39, 1315 (1965).

${ }_{24}$ W. Kemula, S. Brzozowski and K. Butkiewicz, Chemia Anal., Warsaw, 3. 489 (1958).

25 D. Sybilska and A. Bylina, private communication (in press). 